\title{
Posterior Fossa Tumours in the First Year of Life: A Two-Centre Retrospective Study
}

\author{
Stefania Picariello ${ }^{1,2,+}$, Pietro Spennato ${ }^{3,+}{ }^{\oplus}$, Jonathan Roth ${ }^{4}$, Nir Shimony ${ }^{4}{ }^{\oplus}$, Alessandra Marini ${ }^{5}$, \\ Lucia De Martino ${ }^{1}$, Giancarlo Nicosia ${ }^{6}$, Giuseppe Mirone ${ }^{3}$, Maria Serena De Santi ${ }^{3}$, Fabio Savoia ${ }^{7}$, \\ Maria Elena Errico ${ }^{8}$, Lucia Quaglietta ${ }^{1, *}$, Shlomi Costantini ${ }^{4}$ and Giuseppe Cinalli ${ }^{3}$ (i)
}

Citation: Picariello, S.; Spennato, P.; Roth, J.; Shimony, N.; Marini, A.; De Martino, L.; Nicosia, G.; Mirone, G.; De Santi, M.S.; Savoia, F.; et al. Posterior Fossa Tumours in the First Year of Life: A Two-Centre

Retrospective Study. Diagnostics 2022, 12, 635. https://doi.org/10.3390/ diagnostics12030635

Academic Editors:

Angela Mastronuzzi and

Andrea Carai

Received: 7 February 2022

Accepted: 1 March 2022

Published: 4 March 2022

Publisher's Note: MDPI stays neutral with regard to jurisdictional claims in published maps and institutional affiliations.

Copyright: (C) 2022 by the authors. Licensee MDPI, Basel, Switzerland This article is an open access article distributed under the terms and conditions of the Creative Commons Attribution (CC BY) license (https:// creativecommons.org/licenses/by/ $4.0 /)$.
1 Neuro-Oncology Unit, Department of Paediatric Oncology, Santobono-Pausilipon Children's Hospital, Via Mario Fiore 6, 80129 Naples, Italy; s.picariello@santobonopausilipon.it (S.P.); 1.demartino1@santobonopausilipon.it (L.D.M.)

2 Department of Women, Child and General and Specialized Surgery, University of Campania “Luigi Vanvitelli", Via Luigi De Crecchio 4, 80138 Naples, Italy

3 Division of Neurosurgery, Department of Neurosciences, Santobono-Pausilipon Children's Hospital, Via Mario Fiore 6, 80129 Naples, Italy; pierospen@gmail.com (P.S.); peppemirone@hotmail.com (G.M.); serena.desanti@tiscali.it (M.S.D.S.); giuseppe.cinalli@gmail.com (G.C.)

4 Departments of Pediatric Neurosurgery, Dana Children's Hospital, Tel Aviv Medical Center, Tel Aviv University, 6 Weizmann St., Tel Aviv 6423906, Israel; jonaroth@gmail.com (J.R.); nirsh8@gmail.com (N.S.); sconsts@netvision.net (S.C.)

5 Department of Neurosurgery, Ospedale Santa Maria della Misericordia di Perugia, Piazzale Giorgio Menghini 3, 06129 Perugia, Italy; marini.alessandra.am@gmail.com

6 Neurosurgery Unit, San Pio Hospital, Via Pacevecchia 53, 82100 Benevento, Italy; giancarlo.nicosia.gn@gmail.com

7 Childhood Cancer Registry of Campania, Santobono-Pausilipon Children's Hospital, Via della Croce Rossa 8 , 80129 Naples, Italy; f.savoia@santobonopausilipon.it

8 Department of Pathology, Santobono-Pausilipon Children's Hospital, Via Posillipo 226, 80123 Naples, Italy; mariaelenaerrico@virgilio.it

* Correspondence: 1.quaglietta@santobonopausilipon.it; Tel.: +39-081-2205699

+ The authors contributed equally to this work.

\begin{abstract}
Posterior fossa tumours (PFTs) in infants are very rare, and information on these tumours is scarce in the literature. This retrospective study reports their pathological characteristics and describes surgical aspects and treatment outcomes. A two-centre cohort of infants with PFTs treated from 2007 to 2018 was retrospectively reviewed. Patient characteristics, clinical, and treatment data were reviewed. Survival curves for progression-free survival (PFS) and overall survival (OS) were generated. Thirty-three infants were retrieved. There were 11 low grade and 22 high-grade tumours. The most common presenting symptom was intracranial hypertension. Fifteen children out of thirtythree progressed. Five-year PFS was significantly lower in children with high-grade tumours (38.3\%) than those with low-grade tumours $(69.3 \%), p=0.030$. High-grade pathology was the only predictor of progression (HR 3.7, 95\% CI 1.1-13.31), $p=0.045$. Fourteen children with high-grade tumours died, with a 5 -year OS of $55.25 \%$. PFTs in children below one year of age still represent a unique challenge. Infants with high-grade tumours display the worst outcomes and the lowest survival, indicating that more effective strategies are needed.
\end{abstract}

Keywords: infratentorial tumours; posterior fossa tumours; infants; Atypical Teratoid/Rhabdoid Tumour; medulloblastoma; astrocytoma; ependymoma

\section{Introduction}

Primary CNS (Central Nervous System) tumours are the most common solid tumours in children, ranging between $16 \%$ and $23 \%$ of all paediatric malignancies [1-5].

Brain tumours can appear anywhere across the infra and supratentorial compartments, with the posterior fossa being the most common location [3]. In children older than one 
year, over two-thirds of intracranial tumours arise from the cerebellum or brainstem [6,7]. Conversely, the infratentorial location is very rare in children below one year of age; hence relevant aspects of clinical, therapeutic, and biological characterisation are still undefined [8-11].

Signs of raised intracranial pressure, such as macrocephaly, bulging fontanel, and vomit, are the most common onset manifestations, though infants can present with more subtle clinical features, including hypotonia, irritability, poor feeding, failure to thrive, drowsiness, abnormal eye movement, lethargy, and a delay in developmental milestones $[9,12]$. Additional signs and symptoms might be found depending on the tumour location and involvement of anatomical structures and cranial nerves [13,14].

Posterior fossa brain tumours (PFTs) in infancy include a wide range of histopathological types with different biological behaviours and clinical management. Despite the advances in research, providing a more thorough understanding of molecular profiling alongside diagnostic and treatment modalities, the outcome of infants with primary CNS tumours is still poor [15-18].

Surgical resection can be difficult in very young children because of the increased risk of complications-hypotension, hypoxia, hypothermia, and bleeding, associated with the persistence of foetal dural venous circulation pattern and high-grade hypervascular tumours. Not least, the impaired and delayed wound healing together with adjuvant therapy toxicity can greatly contribute to additional morbidity $[19,20]$.

In this 10-year retrospective study, we aimed to provide a description of the clinical and pathologic characteristics of this characteristics of PFTs and to report our experience on management and survival outcomes of infants with posterior fossa tumours.

\section{Materials and Methods}

This is a two-centre retrospective study of infants diagnosed with primitive posterior fossa tumours at the Department of Paediatric Neurosurgery, Santobono-Pausilipon Children's Hospital, Naples, Italy and Dana Children's Hospital, Tel-Aviv Medical Center, Tel Aviv, Israel, between January 2007 and January 2018.

Patients were identified through a review of electronic clinical datasets. Only children with pathology-confirmed tumour diagnosis and available pre and postoperative MRI were included. As per standard clinical practice, all cases were discussed at Neuro-oncology multidisciplinary meetings in both Hospitals.

Demographic and relevant data (presenting symptoms, tumour pathology, surgical management, treatment modalities, tumour recurrence/progression, cause of death) were recorded.

Surgical management of hydrocephalus was classified as external ventricular drainage (EVD), Endoscopic Third Ventriculostomy (ETV), ventriculoperitoneal shunt (VP shunt).

Regarding the surgical approach to the tumour, a prone position with a midline skin incision was adopted in most of the procedures, allowing wide access to the posterior fossa. However, for tumours extending to the cerebellopontine angle, the lateral or park bench position with lateral suboccipital craniotomy was preferred. All patients were transferred post-operatively in the paediatric intensive care unit (PICU) for observation and stabilisation.

Brain MRI with contrast medium administration within $24 \mathrm{~h}$ from surgery was performed to rule out neurosurgical complications and to assess the extent of resection: gross total resection (GTR) (no residual disease), subtotal resection (STR) $(90 \%$ reduction in tumour size), partial resection (50-90\% reduction in tumour size), and biopsy ( $<50 \%$ reduction in tumour size).

Tumour pathology was assigned according to the 2007 and 2016 World Health Organisation Classification of the Central Nervous System, and tumours were grouped into a low grade (0, I and II) and high grade (III and IV) CNS tumours. Metastatic diseases were staged as M0: non metastasis, M1: cells in cerebrospinal fluid (CSF), M2: cranial metastasis, M3: spinal metastasis, and M4: extraneural metastasis. 


\section{Statistical Analysis}

Continuous nonparametric variables are reported as median (range), categorical variables as number and percentage. Differences between groups were compared by Chi-square or Fisher's exact tests for categorical variables and the Mann-Whitney $U$ test for continuous nonparametric variables.

Kaplan-Meier method was used to estimate survival curves for progression-free survival and overall survival. Progression-free survival (PFS) was defined as the time from diagnosis to the first progression, recurrence, or metastatic dissemination. Overall survival (OS) was defined from the diagnosis until death. Patients without events were censored at last follow up or death for PFS. The log-rank test was used to compare survival curves. Cox proportional hazard model was used to estimate hazard ratio and $95 \%$ Confidence Interval (CI) for predictors of progression.

A $p$ value $\leq 0.05$ was considered significant. The software SPSS-IBM 22 was used for all analyses.

\section{Results}

From 2007 to 2018, 33 children under 12 months of age underwent a surgical procedure for a posterior fossa tumour in our Departments (18 at Santobono-Pausilipon Children's Hospital, and 15 at Tel Aviv Medical Center).

The median age at diagnosis was 7.3 (1-12) months, with 15 children (45.5\%) being younger than six months of age. Twenty-three out of thirty-six patients $(70 \%)$ were female.

All children were symptomatic, and none of them had a prenatal diagnosis of PFT. The most common presenting symptom was intracranial hypertension (ICHT) (vomiting, headache, enlarged head circumference, rapid deterioration, sleepiness). It was documented in 27 children out of $33(81.8 \%)$, associated with cerebellar signs (gait disturbances) in four children, torticollis in five, Parinaud's syndrome in three cases, dysphagia in two, strabismus in two, facial nerve palsy in one.

In most cases $(23 / 33,69.7 \%)$, the tumour was located in the midline (cerebellar vermis, brainstem), in two cases in the cerebellar hemisphere (6\%), seven in the cerebellopontine angle $(21.2 \%)$, and one in the pineal region.

CNS WHO grade 0-II tumours were diagnosed in 11 children (33.3\%), whilst highgrade tumours (grade III or IV) in $22(66.7 \%)$. No difference was found in age at diagnosis between children with high-grade and those with low-grade tumours $(p=0.693)$.

Atypical Teratoid/Rhabdoid tumour (AT/RT) was the most common pathology (9, $27 \%)$, followed by pilocytic astrocytoma $(7,21.2 \%)$ and medulloblastomas $(7,21.2 \%)$. Five cases of WHO grade III ependymoma (15.1\%) and one WHO grade II ependymoma were also registered, together with single cases of mature teratoma, desmoplastic infantile glioma, high-grade glioma, and pilomyxoid astrocytoma.

Metastatic disease at diagnosis was documented in three cases of desmoplastic nodular medulloblastoma (2 M2, $1 \mathrm{M} 1)$ and two cases of AT/RT (1 M1, $1 \mathrm{M} 3)$.

\subsection{Management of Hydrocephalus}

Clinical and radiological findings of hydrocephalus were witnessed in 27 patients $(81.8 \%), 9 / 11(81.8 \%)$ children with low-grade pathology and 18/22 (81.8\%) with highgrade tumours $(p=1.000)$.

Surgery for hydrocephalus was carried out in 23 children prior to tumour-directed surgery. In four cases, upfront tumour resection was performed because of the relevant mass effect and neuroradiological evidence of obstructive hydrocephalus.

Ventriculoperitoneal (VP) shunt was implanted in 14 children (42.4\%), EVD was initially preferred in 4 patients, associated with ETV in 2 cases. Endoscopic third ventriculostomy was performed at admission in further five patients, in association with Ommaya reservoir in two of them.

However, four out of seven children treated with ETV experienced ETV failure and persistent hydrocephalus after tumour resection and required a VP shunt placement. 


\subsection{Survival Outcome}

Patients were followed for a median follow up time of 43 (1-117) months.

Fifteen children out of 33 progressed with a whole population 5-year (60 months) PFS of $49.2 \%$ (Figure 1 ).

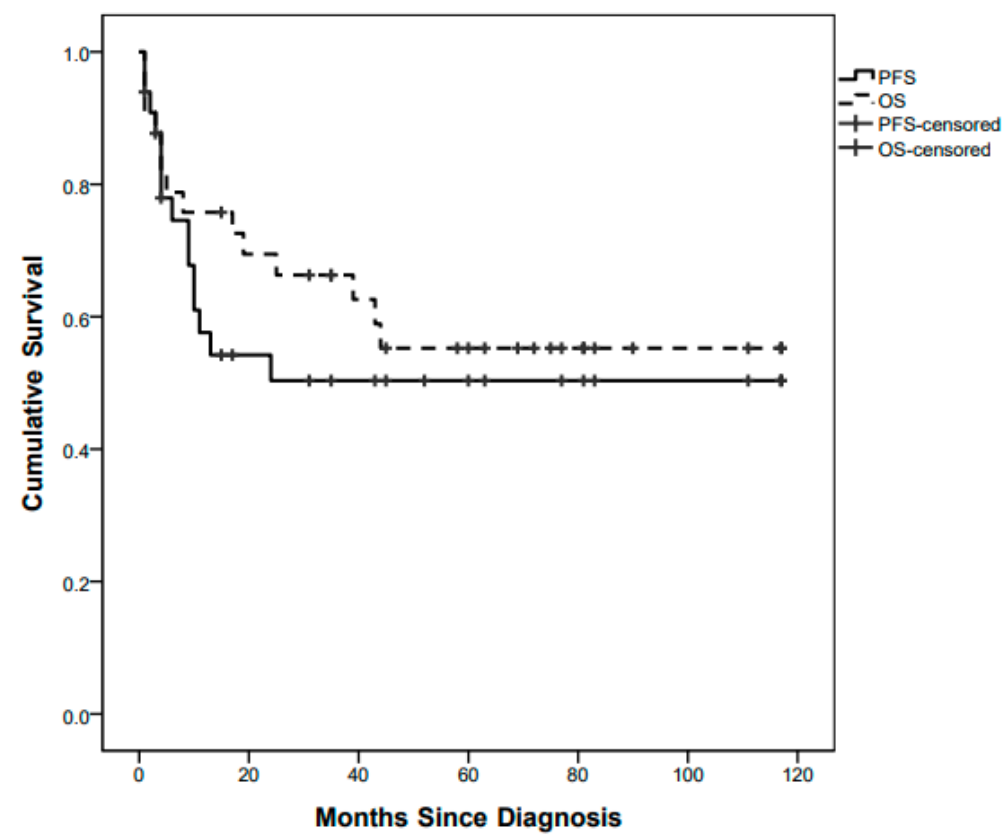

Figure 1. Whole-population Progression-Free Survival (PFS) and Overall Survival (OS) curves.

PFS curves were significantly different between children with low-grade tumours (3/11 events, 5-year PFS 69.3\%) and those with high-grade tumours (12/22 events, 5-year PFS 38.3\%), $p=0.030$ (Figure 2).

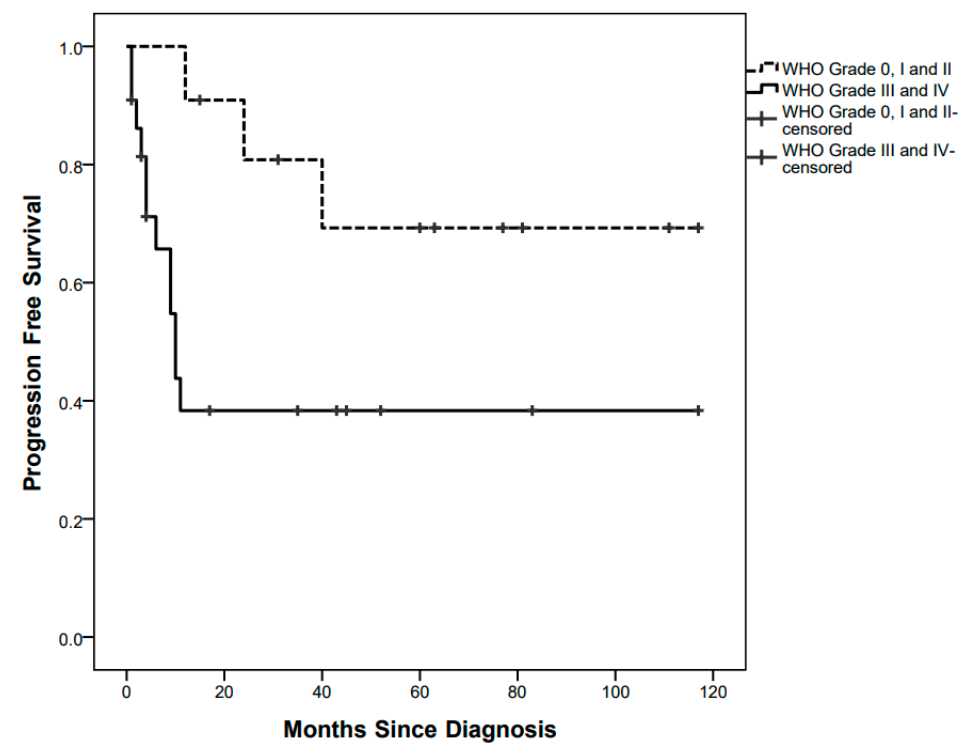

Figure 2. Progression-Free Survival curves by low-grade and high-grade pathology.

High-grade pathology was indeed the only predictor of progression in the univariate Cox Regression analysis (HR 3.7, 95\% CI 1.1-13.31, $p=0.045$ ), while the other explored variables were not found statistically significant (ICHT $p=0.411$; age at diagnosis $p=0.115$; GTR $p=0.165)$. 
Fourteen children died after a median of 6.5 (1-44) months since diagnosis, at a median age of 13 (2-56) months, with a 5-year OS of 55.25\%. All of them were affected by high-grade tumours (Figure 3).

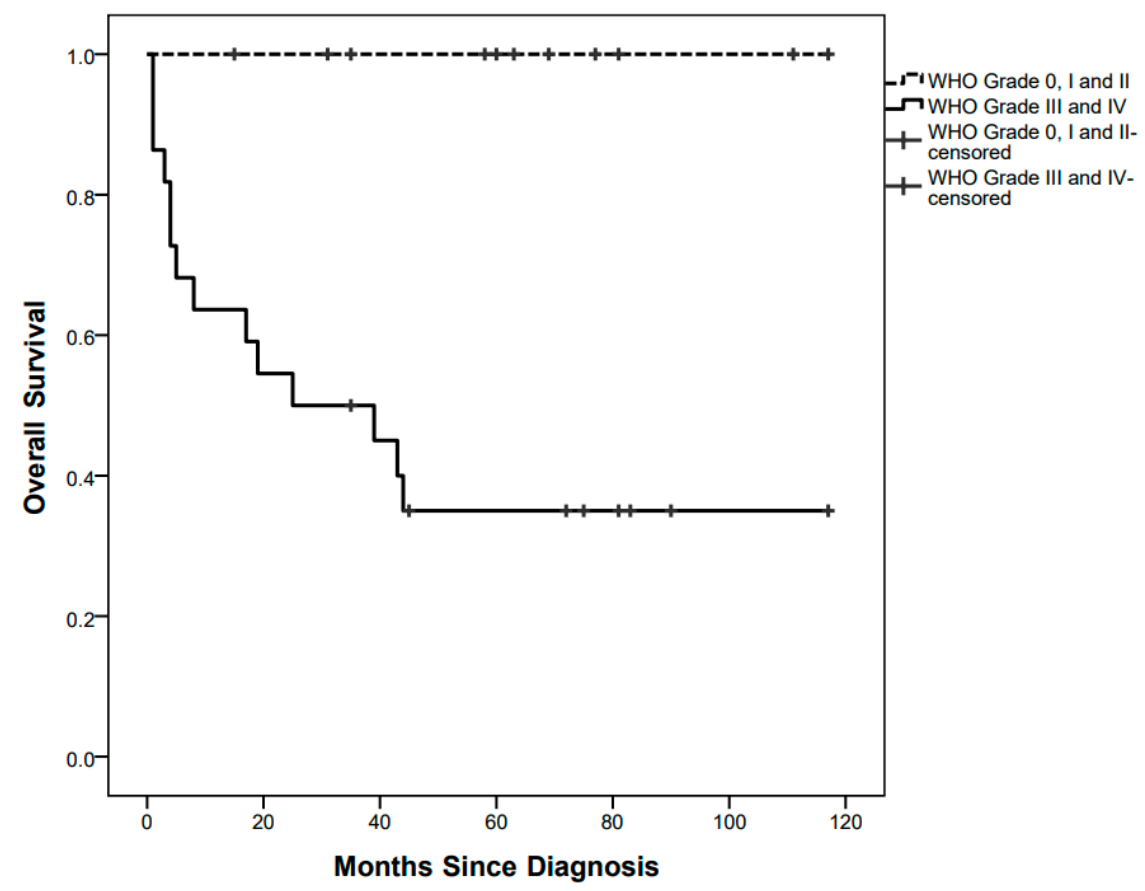

Figure 3. Overall survival curves by low grade and high-grade pathology.

\subsection{Treatment Details and Outcome Stratified by WHO Grade}

\subsubsection{Grade 0 (One Patient)}

A pineal mature teratoma underwent subtotal resection and did not recur over more than five years of follow up.

\subsubsection{Grade I (Eight Patients)}

Seven grade I tumours were pilocytic astrocytomas. GTR was obtained in five of them, STR in one, one child had brainstem invasion requiring a more conservative approach (partial removal) followed by standard Vincristine-Carboplatin chemotherapy treatment [21]. The latter progressed 24 months after diagnosis and was completely resected. Lastly, a desmoplastic infantile glioma of the brainstem was subjected to biopsy at diagnosis and underwent a watch and wait strategy with stable disease 15 months after diagnosis.

\subsubsection{Grade II (Two Patients)}

One case of WHO grade II Ependymoma was treated as per SIOP Ependymoma I Protocol [22]. A subtotal resection was achieved, but the residual lesion progressed 12 months later, and the child was treated with GTR followed by radiotherapy. The child was alive with no evidence of disease at 35 months of follow-up.

The second child had an exophytic brainstem pilomyxoid astrocytoma and underwent subtotal resection and chemotherapy according to Vincristine-Carboplatin chemotherapy treatment [21]. Recurrence occurred 40 months later and was treated with surgery and second-line chemotherapy with no further progression at 69 months of follow-up.

\subsubsection{Grade III (Five Patients)}

All these patients had anaplastic ependymomas. Three children were treated with GTR resection and chemotherapy, per Italian protocol for paediatric intracranial ependymoma [23]. All children relapsed during follow-up: two patients progressed at 9 and 
17 months after diagnosis and died during their fourth year of follow-up. One child progressed 22 months after diagnosis and was alive with no evidence of disease at 52 months of follow-up after surgical resection of a single lumbar metastasis followed by focal radiotherapy (Figure 4).

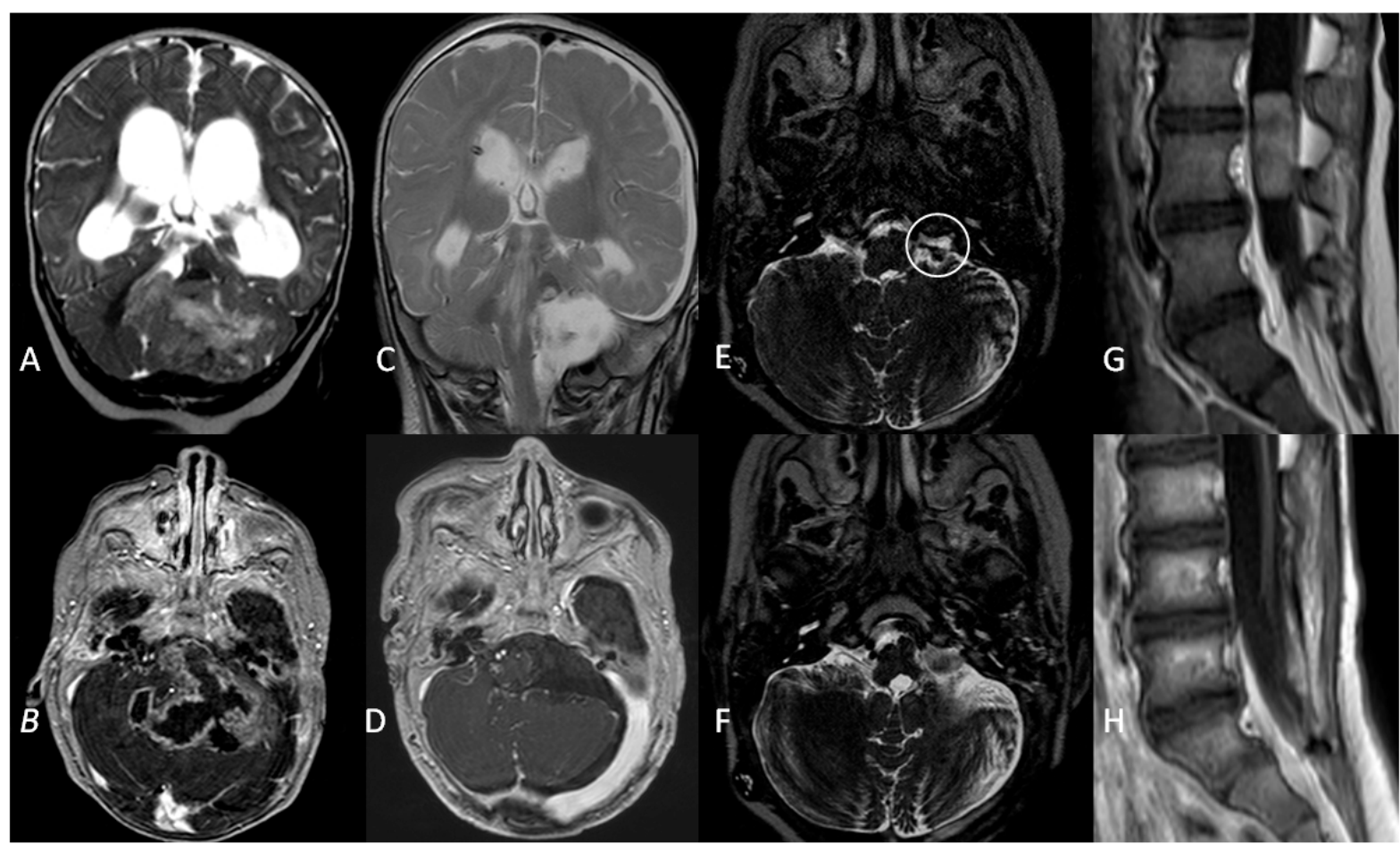

Figure 4. A coronal T2W MRI (A) and axial contrast-enhanced T1 W MRI (B) showing a posterior fossa tumour involving the left cerebellopontine angle and extending into the fourth ventricle in a 5-month old infant. (C,D) Three-month follow-up MRI showing complete surgical resection and VP shunt. (E). An axial T2W MRI at 1-year follow-up showing recurrence (white circle) of the tumour in the left lateral recess. (F). Gross total resection of the tumour recurrence. (G). Metastatic dissemination 22 months after diagnosis (single lumbar metastasis). (H). Complete removal of the spinal lesion.

Two children were treated per SIOP Ependymoma I Protocol [22]. The former had metastatic spinal dissemination six months after the GTR surgery and was treated with extensive surgical removal, radiotherapy, and metronomic treatment. Unfortunately, the child died after two years of follow-up because of multiple disease progressions. A GTR was initially achieved in the latter, with subsequent tumour progression at 10 months. A second surgical excision was attempted (GTR) followed by radiotherapy with a stable remnant lesion at 81 months.

\subsubsection{Grade IV (17 Patients)}

Nine grade IV patients were affected by AT/RT, metastatic in two cases (one M1, one M3); all of them underwent a surgical procedure (two GTR, two STR, four partial resection and one biopsy) followed by adjuvant therapy based on European Rhabdoid Registry consensus therapy recommendation [24]. Two patients only survived during the follow-up.

Seven patients had a diagnosis of medulloblastoma, namely large cell medulloblastoma in one case and desmoplastic/nodular pathology in six cases, with cranial metastases in two. The former underwent a biopsy and died of gastro-intestinal haemorrhage due to acute chemotherapy toxicity two months later. The other patients had surgery (one partial removal and five GTR) and induction chemotherapy followed by high dose myeloablative chemotherapy with autologous stem cell rescue (HDC/ASCR). Two of them died of complications several months after treatment: one patient developed chronic renal and cardiac 
failure associated with myelodysplasia, resulting in hemocoagulative disorders. She also experienced multiple episodes of shunt failure and a mycotic ventriculitis and eventually died of massive intraventricular haemorrhage (Figure 5).

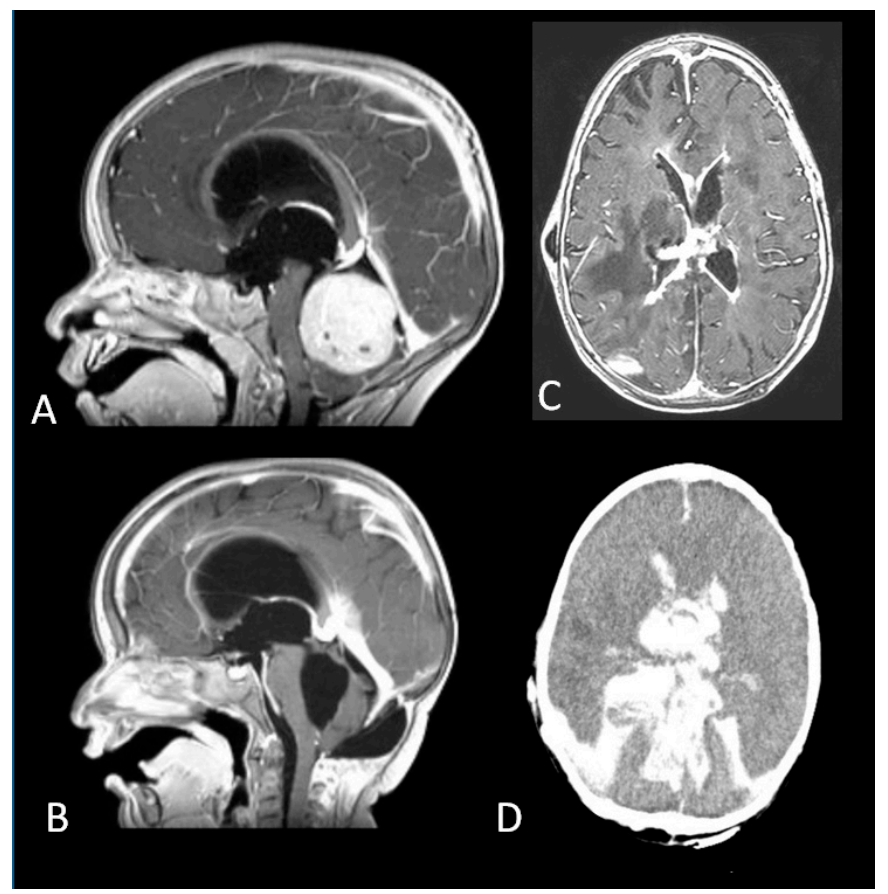

Figure 5. (A). A fourth ventricular tumour arising from the cerebellar vermis. (B). Complete Resection of a desmoplastic medulloblastoma. (C). Mycotic ventriculitis (D). Intraventricular haemorrhage.

The second patient experienced cardiac failure and died when she was on mechanical circulatory support as a bridge to a heart transplant.

One child died of acute toxicity three months after diagnosis due to acute renal failure following the second cycle of chemotherapy.

The patient with high-grade glioma had a partial removal and induction chemotherapy followed by HDC/ ASCR and focal radiotherapy. The child progressed two months after the end of treatment and was treated with Temozolomide and Bevacizumab, interrupted after the first cycle due to cardiotoxicity. Cyberknife radiosurgery was therefore performed. The child is now stable after six years of follow-up.

\section{Discussion}

Brain tumours in the first year of life differ in topographical distribution, tumour biology, clinical course, and prognosis compared with those diagnosed at older ages [16,25].

The prognosis is usually poor in infants, and the outcome is largely related to pathological and biological characteristics, size, and the location of the tumour [26-28].

This is extremely relevant to tumours involving the vital brainstem nuclei and critical cerebellar structures $[29,30]$. The posterior fossa is indeed the commonest site of primary brain tumours in older children, whilst the supratentorial location is more prevalent in those below one year of age, infratentorial tumours accounting for $17.9-32.4 \%$ depending on series [8,31].

In view of their rarity, posterior fossa tumours presenting in the first year of life still represent a unique challenge, and a focus of intense research aimed not just at prolonging survival but also at improving the quality of survival [11,32-35].

In this multicentre study, we focus on the clinical presentation, the histopathological diagnosis, the surgical management of hydrocephalus, treatment, and survival outcome of 33 infants with posterior fossa tumours diagnosed and followed over a decade. 
Clinical and radiological findings of hydrocephalus were documented in more than $80 \%$ of children at the time of presentation in our series, in line with previous literature $[9,36,37]$.

In our centres, CSF diversion is performed before tumour resection surgery in most infant cases, with the aim of relieving the intracranial pressure and facilitating the tumour resection. External ventricular drain placement is performed in the presence of acutely deteriorating consciousness and cases requiring prompt stabilisation, though close clinical monitoring is required afterwards to avoid overdrainage and infective complications. Alternatively, a VP shunt can be placed, particularly in cases with the metastatic or multicentric disease, whilst ETV is a valid option to temporarily control the hydrocephalus, avoiding EVD or permanent VP shunt [19,38-40].

To date, there is no consensus on the best management strategy of hydrocephalus in children with posterior fossa tumours, particularly in terms of the timing of the CSF diversion and the indications and efficacy of ETV and VP shunt in different clinical scenarios. The choice between the surgical technique is often dictated by the severity of symptoms and the degree of the hydrocephalus, together with the personal choice of the surgeon $[36,41,42]$.

The majority of children with PFT-related ICHT usually experience resolution after removal of the underlying disease, whilst about $10-40 \%$ have persistent hydrocephalus. Several risk factors associated with permanent CSF diversion in children with PFT have been identified in previous studies, namely age $<2-3$ years at surgery, perioperative EVD placement, postresection complications and signs of hydrocephalus in postoperative imaging, tumour type and location, metastatic dissemination, and subtotal tumour resection [43-45].

Some authors recommend preresection ETV in patients with a high risk of persisting postoperative hydrocephalus and in patients whose tumour surgery is delayed. In fact, despite ETV failure occurring sooner than VP shunt failure, VP shunt placement is burdened with a higher rate of postoperative complications and VP shunt revision surgeries that might delay the adjuvant treatment in the early treatment phase or dramatically affect the quality of life in the long-term survivors [38,46-49].

However, in our cohort of high-risk infants, ETV was associated with a high incidence of failure and over half of the patients eventually required VP shunt despite tumour resection. Hence, the optimal method of CSF diversion in infants with PFT remains largely debated, and hydrocephalus management requires an individualised case-based approach.

In infants with brain tumours, tumour location is correlated with the outcome, too. In fact, supratentorial tumours are usually low grade in contrast to their infratentorial counterparts, which are malignant in most cases [8,9].

In our series, the prevalence of high-grade tumours was double that of the low-grade ones. AT/RT was the most common pathology, accounting for a quarter of the study cohort. Allowing for tumour location, this particular finding is in contrast with previous literature, reporting teratoma and pilocytic astrocytoma as the most common pathology in infants, followed by medulloblastoma [6,50,51].

Besides WHO grade, we failed to identify any predictors of survival outcome. In fact, the small number of patients included in the study, together with the heterogeneity of histopathology and the variety of treatment strategies adopted, preclude any further and detailed analysis.

However, it is worthwhile to note that, despite young age being a risk factor for progression and death, none of the children with low-grade tumours died. In contrast, almost two-thirds of children with high-grade tumours experienced progression and eventually died. Among deceased subjects, over half died within six months after diagnosis, and four children affected by medulloblastoma died of treatment-related toxicity.

There is no current guideline for medulloblastoma under the age of 36 months. A trial is being developed by SIOP but has not yet opened. Multiple treatment strategies have been adopted in the treatment of very young children with posterior fossa tumours, 
yielding extremely variable results and still dismal prognosis, particularly for high-grade tumours $[35,52,53]$.

Surgery is the mainstay of treatment for brain tumours in the first year of life, and the extent of resection is strictly related to the outcome, while radiotherapy is usually avoided in infants due to the detrimental effect on the immature and growing brain [54]. However, gross total resection is not always feasible and often encumbered by a number of immediate postsurgical complications and a higher mortality rate compared with older children [31]. High-grade pathology additionally raises the risk of postoperative complications, resulting in even higher and sobering figures [55,56]. Standard dose chemotherapy and HDC/ASCR are widely recognised as effective adjuvant treatments, particularly in cases with malignant pathology, and play an important role in delaying radiotherapy at a later stage in children with residual or recurrent disease. Despite these advantages, chemotherapy itself can be a cause of life-threatening side effects and contribute to both early and delayed mortality. Moreover, such a fragile population of young children can suffer from several late effects and long-term disability brought on by the tumour as well as the treatment strategies [57-62].

That is why advances in local surgical treatments and a better understanding of tumour molecular biology, along with the development of targeted therapies, are indispensable to provide risk stratification and to improve the now scarce chance of cure.

There are limitations to the present study that should be acknowledged owing to its retrospective design. First, beyond survival, the heterogeneity of tumours and the small numbers did not allow us to perform a detailed analysis of prognostic factors in this highly selected cohort of infants. Secondly, postoperative complications, long-term sequelae, and functional outcomes are not reported in the present study. However, the short and long-term morbidity in survivors, caused by the disease itself and the detrimental effect of multiple treatment modalities, is particularly relevant in children below one year of age and should be acknowledged [32,55-63].

Despite these limitations, we provide a large descriptive cohort of posterior fossa tumours presenting in infancy. These data raise awareness of the need for further research and implementation of current knowledge and clinical practice.

\section{Conclusions}

Posterior fossa tumours in children below one year old still represent a unique challenge because of their aggressive behaviour and the scarce chance of cure. The prognosis is poor and closely dependent on the histopathological grade and tumour type. Infants with high-grade tumours display the worst outcomes and the lowest overall survival, whilst those with low-grade tumours inevitably present long-term disability.

Surgery continues to be the mainstay of treatment, albeit local salvage therapy contributes to morbidity.

Efforts focused on providing advances in surgical techniques and improving molecular understanding of tumour biology are crucial in this particularly vulnerable population in order to improve the chance of cure and the long-term outcomes.

Author Contributions: Conceptualization, S.P., P.S. and G.C.; methodology, S.P., P.S. and G.C.; formal analysis, S.P. and P.S.; data curation, A.M., N.S., J.R., F.S., L.D.M. and M.E.E.; writing-original draft preparation, S.P. and P.S.; visualisation: G.N., G.M. and M.S.D.S.; writing-review \& editing, S.P., J.R., G.C. and L.Q.; supervision, S.C. and G.C. All authors have read and agreed to the published version of the manuscript.

Funding: This research received no external funding.

Institutional Review Board Statement: This retrospective study was conducted in accordance with the standards as laid down in the 1964 Declaration of Helsinki and its later amendments or comparable ethical standards. For this type of study, ethical approval was not required since it was carried out as an internal audit conducted within the two hospitals. 
Informed Consent Statement: Written informed consent was obtained from all subjects involved in the study.

Data Availability Statement: The data presented in this study are available on request from the corresponding author.

Conflicts of Interest: The authors declare no conflict of interest.

\section{References}

1. Stiller, C.A.; Bayne, A.M.; Chakrabarty, A.; Kenny, T.; Chumas, P. Incidence of childhood CNS tumours in Britain and variation in rates by definition of malignant behaviour: Population-based study. BMC Cancer 2019, 19, 139. [CrossRef] [PubMed]

2. Williams, T.; Naushahi, M.; Bernard, A.; Campbell, R. Epidemiology of paediatric central nervous system tumours in Queensland, Australia. J. Clin. Neurosci. 2021, 92, 126-130. [CrossRef] [PubMed]

3. Ostrom, Q.T.; Cioffi, G.; Waite, K.; Kruchko, C.; Barnholtz-Sloan, J.S. CBTRUS Statistical Report: Primary Brain and Other Central Nervous System Tumors Diagnosed in the United States in 2014-2018. Neuro-Oncology 2021, 23, iii1-iii105. [CrossRef]

4. Lannering, B.; Sandström, P.-E.; Holm, S.; Lundgren, J.; Pfeifer, S.; Samuelsson, U.; Strömberg, B.; Gustafsson, G.; Swedish Childhood CNS Tumor Working Group (VCTB). Classification, incidence and survival analyses of children with CNS tumours diagnosed in Sweden 1984-2005. Acta Paediatr. 2009, 98, 1620-1627. [CrossRef] [PubMed]

5. Arora, R.S.; Alston, R.D.; Eden, T.O.B.; Estlin, E.J.; Moran, A.; Birch, J.M. Age-incidence patterns of primary CNS tumors in children, adolescents, and adults in England. Neuro-Oncology 2009, 11, 403-413. [CrossRef]

6. Bishop, A.J.; McDonald, M.W.; Chang, A.L.; Esiashvili, N. Infant brain tumors: Incidence, survival, and the role of radiation based on Surveillance, Epidemiology, and End Results (SEER) Data. Int. J. Radiat. Oncol. Biol. Phys. 2012, 82, 341-347. [CrossRef]

7. Alves, C.A.P.F.; Löbel, U.; Martin-Saavedra, J.S.; Toescu, S.; Tsunemi, M.H.; Teixeira, S.R.; Mankad, K.; Hargrave, D.; Jacques, T.S.; da Costa Leite, C.; et al. A diagnostic algorithm for posterior fossa tumors in children: A validation study. AJNR Am. J. Neuroradiol. 2021, 42, 961-968. [CrossRef]

8. Al-Hussaini, M.; Swaidan, M.; Al-Jumaily, U.; Musharbash, A. Central nervous system tumors in the first year of life: A clinical and pathologic experience from a single cancer center. Childs Nerv. Syst. 2013, 29, 1883-1891. [CrossRef]

9. Mehrotra, N.; Shamji, M.F.; Vassilyadi, M.; Ventureyra, E.C.G. Intracranial tumors in first year of life: The CHEO experience. Childs Nerv. Syst. 2009, 25, 1563-1569. [CrossRef]

10. Ceglie, G.; Vinci, M.; Carai, A.; Rossi, S.; Colafati, G.S.; Cacchione, A.; Tornesello, A.; Miele, E.; Locatelli, F.; Mastronuzzi, A. Infantile/Congenital High-Grade Gliomas: Molecular Features and Therapeutic Perspectives. Diagnostics 2020, 10, 648. [CrossRef]

11. Simone, V.; Rizzo, D.; Cocciolo, A.; Caroleo, A.M.; Carai, A.; Mastronuzzi, A.; Tornesello, A. Infantile brain tumors: A review of literature and future perspectives. Diagnostics 2021, 11, 670. [CrossRef] [PubMed]

12. Jaing, T.-H.; Wu, C.-T.; Chen, S.-H.; Hung, P.-C.; Lin, K.-L.; Jung, S.-M.; Tseng, C.-K. Intracranial tumors in infants: A single institution experience of 22 patients. Childs Nerv. Syst. 2011, 27, 415-419. [CrossRef] [PubMed]

13. Goethe, E.A.; Gadgil, N.; Stormes, K.; Wassef, A.; LoPresti, M.; Lam, S. Predicting dysphagia in children undergoing surgery for posterior fossa tumors. Childs Nerv. Syst. 2020, 36, 925-931. [CrossRef]

14. Fąfara-Leś, A.; Kwiatkowski, S.; Maryńczak, L.; Kawecki, Z.; Adamek, D.; Herman-Sucharska, I.; Kobylarz, K. Torticollis as a first sign of posterior fossa and cervical spinal cord tumors in children. Childs Nerv. Syst. 2014, 30, 425-430. [CrossRef] [PubMed]

15. Hicks, D.; Rafiee, G.; Schwalbe, E.C.; Howell, C.I.; Lindsey, J.C.; Hill, R.M.; Smith, A.J.; Adidharma, P.; Steel, C.; Richardson, S.; et al. The molecular landscape and associated clinical experience in infant medulloblastoma: Prognostic significance of second-generation subtypes. Neuropathol. Appl. Neurobiol. 2021, 47, 236-250. [CrossRef]

16. Lu, V.M.; Di, L.; Eichberg, D.G.; Luther, E.M.; Shah, A.H.; Daniels, D.J.; Maher, O.M.; Niazi, T.N. Age of diagnosis clinically differentiates Atypical Teratoid/Rhabdoid Tumors diagnosed below age of 3 years: A database study. Childs Nerv. Syst. 2021, 37, 1077-1085. [CrossRef] [PubMed]

17. Hill, R.M.; Richardson, S.; Schwalbe, E.C.; Hicks, D.; Lindsey, J.C.; Crosier, S.; Rafiee, G.; Grabovska, Y.; Wharton, S.B.; Jacques, T.S.; et al. Time, pattern, and outcome of medulloblastoma relapse and their association with tumour biology at diagnosis and therapy: A multicentre cohort study. Lancet Child Adolesc. Health 2020, 4, 865-874. [CrossRef]

18. Faltermeier, C.; Chai, T.; Syed, S.; Lau, N.; Elkaim, L.; Ibrahim, G.; Wang, A.; Weil, A.; Bendel, A.; Fallah, A.; et al. Survival of infants $\leq 24$ months of age with brain tumors: A population-based study using the SEER database. PLoS ONE 2019, 14, e0223051. [CrossRef]

19. Spennato, P.; Nicosia, G.; Quaglietta, L.; Donofrio, V.; Mirone, G.; Di Martino, G.; Guadagno, E.; del Basso de Caro, M.L.; Cascone, D.; Cinalli, G. Posterior fossa tumors in infants and neonates. Childs Nerv. Syst. 2015, 31, 1751-1772. [CrossRef]

20. Klein, O.; Boussard, N.; Guerbouz, R.; Helleringer, M.; Joud, A.; Puget, S. Surgical approach to the posterior fossa in children, including anesthetic considerations and complications: The prone and the sitting position. Technical note. Neurochirurgie 2021, 67, 46-51. [CrossRef]

21. Gnekow, A.K.; Walker, D.A.; Kandels, D.; Picton, S.; Perilongo, G.; Grill, J.; Stokland, T.; Sandstrom, P.E.; Warmuth-Metz, M.; Pietsch, T.; et al. A European randomised controlled trial of the addition of etoposide to standard vincristine and carboplatin induction as part of an 18-month treatment programme for childhood ( $\leq 16$ years) low grade glioma-A final report. Eur. J. Cancer 2017, 81, 206-225. [CrossRef] [PubMed] 
22. Ritzmann, T.A.; Chapman, R.J.; Kilday, J.-P.; Thorp, N.; Modena, P.; Dineen, R.A.; Macarthur, D.; Mallucci, C.; Jaspan, T.; Pajtler, K.W.; et al. SIOP Ependymoma I: Final results, long term follow-up and molecular analysis of the trial cohort: A BIOMECA Consortium Study. Neuro-Oncology, 2022; Online ahead of print. [CrossRef] [PubMed]

23. Massimino, M.; Miceli, R.; Giangaspero, F.; Boschetti, L.; Modena, P.; Antonelli, M.; Ferroli, P.; Bertin, D.; Pecori, E.; Valentini, L.; et al. Final results of the second prospective AIEOP protocol for pediatric intracranial ependymoma. Neuro-Oncology 2016, 18, 1451-1460. [CrossRef] [PubMed]

24. Seeringer, A.; Bartelheim, K.; Kerl, K.; Hasselblatt, M.; Leuschner, I.; Rutkowski, S.; Timmermann, B.; Kortmann, R.D.; Koscielniak, E.; Schneppenheim, R.; et al. Feasibility of intensive multimodal therapy in infants affected by rhabdoid tumors-Experience of the EU-RHAB registry. Klin. Padiatr. 2014, 226, 143-148. [CrossRef]

25. Rivera-Luna, R.; Medina-Sanson, A.; Leal-Leal, C.; Pantoja-Guillen, F.; Zapata-Tarrés, M.; Cardenas-Cardos, R.; Barrera-Gómez, R.; Rueda-Franco, F. Brain tumors in children under 1 year of age: Emphasis on the relationship of prognostic factors. Childs Nerv. Syst. 2003, 19, 311-314. [CrossRef]

26. Lopez-Rivera, V.; Dono, A.; Abdelkhaleq, R.; Sheth, S.A.; Chen, P.R.; Chandra, A.; Ballester, L.Y.; Esquenazi, Y. Treatment trends and overall survival in patients with grade II/III ependymoma: The role of tumor grade and location. Clin. Neurol. Neurosurg. 2020, 199, 106282. [CrossRef]

27. Aras, Y.; Dölen, D.; İribasÇelik, A.; Kılıç, G.; Kebudi, R.; Ünverengil, G.; Sabancı, P.A.; İzgi, A.N. Effects of different molecular subtypes and tumor biology on the prognosis of medulloblastoma. Childs Nerv. Syst. 2021, 37, 3733-3742. [CrossRef]

28. Qin, Q.; Huang, D.; Jiang, Y. Survival difference between brainstem and cerebellum medulloblastoma: The surveillance, epidemiology, and end results-based study. Medicine 2020, 99, e22366. [CrossRef]

29. Klimo, P.; Nesvick, C.L.; Broniscer, A.; Orr, B.A.; Choudhri, A.F. Malignant brainstem tumors in children, excluding diffuse intrinsic pontine gliomas. J. Neurosurg. Pediatr. 2016, 17, 57-65. [CrossRef]

30. Totapally, B.R.; Shah, A.H.; Niazi, T. Epidemiology and short-term surgical outcomes of children presenting with cerebellar tumors. Clin. Neurol. Neurosurg. 2018, 168, 97-101. [CrossRef]

31. Toescu, S.M.; James, G.; Phipps, K.; Jeelani, O.; Thompson, D.; Hayward, R.; Aquilina, K. Intracranial neoplasms in the first year of life: Results of a third cohort of patients from a single institution. Neurosurgery 2019, 84, 636-646. [CrossRef] [PubMed]

32. Lassaletta, A.; Bouffet, E.; Mabbott, D.; Kulkarni, A.V. Functional and neuropsychological late outcomes in posterior fossa tumors in children. Childs Nerv. Syst. 2015, 31, 1877-1890. [CrossRef] [PubMed]

33. Charalambides, C.; Dinopoulos, A.; Sgouros, S. Neuropsychological sequelae and quality of life following treatment of posterior fossa ependymomas in children. Childs Nerv. Syst. 2009, 25, 1313-1320. [CrossRef] [PubMed]

34. Lassaletta, A. Medulloblastoma in infants: The never-ending challenge. Lancet Oncol. 2018, 19, 720-721. [CrossRef]

35. Baliga, S.; Gandola, L.; Timmermann, B.; Gail, H.; Padovani, L.; Janssens, G.O.; Yock, T.I. Brain tumors: Medulloblastoma, ATRT, ependymoma. Pediatr. Blood Cancer 2021, 68 (Suppl. 2), e28395. [CrossRef]

36. Due-Tønnessen, B.J.; Helseth, E. Management of hydrocephalus in children with posterior fossa tumors: Role of tumor surgery. Pediatr. Neurosurg. 2007, 43, 92-96. [CrossRef]

37. Sainte-Rose, C.; Cinalli, G.; Roux, F.E.; Maixner, R.; Chumas, P.D.; Mansour, M.; Carpentier, A.; Bourgeois, M.; Zerah, M.; Pierre-Kahn, A.; et al. Management of hydrocephalus in pediatric patients with posterior fossa tumors: The role of endoscopic third ventriculostomy. J. Neurosurg. 2001, 95, 791-797. [CrossRef]

38. Ruggiero, C.; Cinalli, G.; Spennato, P.; Aliberti, F.; Cianciulli, E.; Trischitta, V.; Maggi, G. Endoscopic third ventriculostomy in the treatment of hydrocephalus in posterior fossa tumors in children. Childs Nerv. Syst. 2004, 20, 828-833. [CrossRef]

39. Habib, H.A.M. Intraoperative precautionary insertion of external ventricular drainage catheters in posterior fossa tumors presenting with hydrocephalus. Alex. J. Med. 2014, 50, 333-340. [CrossRef]

40. El-Gaidi, M.A.; El-Nasr, A.H.A.; Eissa, E.M. Infratentorial complications following preresection CSF diversion in children with posterior fossa tumors. J. Neurosurg. Pediatr. 2015, 15, 4-11. [CrossRef]

41. Kirkman, M.A.; Hayward, R.; Phipps, K.; Aquilina, K. Surgical decision-making in the management of childhood tumors of the CNS disseminated at presentation. J. Neurosurg. Pediatr. 2018, 21, 563-573. [CrossRef] [PubMed]

42. Elkatatny, A.M.; Zurakowski, D.; Goumnerova, L. 352 management strategies for hydrocephalus in pediatric posterior fossa brain tumor. Neurosurgery 2018, 65, 140. [CrossRef]

43. Helmbold, L.J.; Kammler, G.; Regelsberger, J.; Fritzsche, F.S.; Emami, P.; Schüller, U.; Krajewski, K. Predictive factors associated with ventriculoperitoneal shunting after posterior fossa tumor surgery in children. Childs Nerv. Syst. 2019, 35, 779-788. [CrossRef]

44. Pilotto, C.; Liguoro, I.; Scaravetti, S.; Passone, E.; D’Agostini, S.; Tuniz, F.; Skrap, M.; Cogo, P. Risk Factors of Persistent Hydrocephalus in Children with Brain Tumor: A Retrospective Analysis. Pediatr. Neurosurg. 2021, 56, 205-212. [CrossRef] [PubMed]

45. Pitsika, M.; Fletcher, J.; Coulter, I.C.; Cowie, C.J.A. A validation study of the modified Canadian Preoperative Prediction Rule for Hydrocephalus in children with posterior fossa tumors. J. Neurosurg. Pediatr. 2021, 28, 121-127. [CrossRef]

46. Paunović, A.; Milisavljević, F.; Bošković, J. Evaluation of clinical characteristics as indicators for shunt procedure in patients with medulloblastoma: PS210. Porto Biomed. J. 2017, 2, 240-241. [CrossRef]

47. Lin, C.-T.; Riva-Cambrin, J.K. Management of posterior fossa tumors and hydrocephalus in children: A review. Childs Nerv. Syst. 2015, 31, 1781-1789. [CrossRef] [PubMed] 
48. Ben Zvi, I.; Adegboyega, G.; Toescu, S.M.; Schwartz, N.; Kershenovich, A.; Toledano, H.; Michowiz, S.; Aquilina, K. Postoperative hydrocephalus management may cause delays in adjuvant treatment following paediatric posterior fossa tumour resection: A multicentre retrospective observational study. Childs Nerv. Syst. 2022, 38, 311-317. [CrossRef]

49. Foreman, P.; McClugage, S.; Naftel, R.; Griessenauer, C.J.; Ditty, B.J.; Agee, B.S.; Riva-Cambrin, J.; Wellons, J. Validation and modification of a predictive model of postresection hydrocephalus in pediatric patients with posterior fossa tumors. J. Neurosurg. Pediatr. 2013, 12, 220-226. [CrossRef]

50. Ghodsi, S.M.; Habibi, Z.; Hanaei, S.; Moradi, E.; Nejat, F. Brain tumors in infants. J. Pediatr. Neurosci. 2015, 10, 335-340. [CrossRef]

51. Larouche, V.; Huang, A.; Bartels, U.; Bouffet, E. Tumors of the central nervous system in the first year of life. Pediatr. Blood Cancer 2007, 49, 1074-1082. [CrossRef] [PubMed]

52. Dhall, G.; O'Neil, S.H.; Ji, L.; Haley, K.; Whitaker, A.M.; Nelson, M.D.; Gilles, F.; Gardner, S.L.; Allen, J.C.; Cornelius, A.S.; et al. Excellent outcome of young children with nodular desmoplasticmedulloblastoma treated on "Head Start" III: A multi-institutional, prospective clinical trial. Neuro-Oncology 2020, 22, 1862-1872. [CrossRef] [PubMed]

53. Benesch, M.; Bartelheim, K.; Fleischhack, G.; Gruhn, B.; Schlegel, P.G.; Witt, O.; Stachel, K.D.; Hauch, H.; Urban, C.; Quehenberger, F.; et al. High-dose chemotherapy (HDCT) with auto-SCT in children with Atypical Teratoid/Rhabdoid Tumors (AT/RT): A report from the European Rhabdoid Registry (EU-RHAB). Bone Marrow Transplant. 2014, 49, 370-375. [CrossRef] [PubMed]

54. Shen, C.J.; Perkins, S.M.; Bradley, J.A.; Mahajan, A.; Marcus, K.J. Radiation therapy for infants with cancer. Pediatr. Blood Cancer 2021, 68 (Suppl. 2), e28700. [CrossRef] [PubMed]

55. Tartaglione, T.; Izzo, G.; Alexandre, A.; Botto, A.; Di Lella, G.M.; Gaudino, S.; Caldarelli, M.; Colosimo, C. MRI findings of olivary degeneration after surgery for posterior fossa tumours in children: Incidence, time course and correlation with tumour grading. Radiol. Med. 2015, 120, 474-482. [CrossRef]

56. Grønbæk, J.K.; Wibroe, M.; Toescu, S.; Frič, R.; Thomsen, B.L.; Møller, L.N.; Grillner, P.; Gustavsson, B.; Mallucci, C.; Aquilina, K.; et al. Postoperative speech impairment and surgical approach to posterior fossa tumours in children: A prospective European multicentre cohort study. Lancet Child. Adolesc. Health 2021, 5, 814-824. [CrossRef]

57. Kulkarni, A.V.; Piscione, J.; Shams, I.; Bouffet, E. Long-term quality of life in children treated for posterior fossa brain tumors. J. Neurosurg. Pediatr. 2013, 12, 235-240. [CrossRef]

58. Chieffo, D.P.R.; Arcangeli, V.; Moriconi, F.; Zanetti, C.; Frassanito, P.; Bianchi, F.; Massimi, L.; Tamburrini, G. Correlation between Pre- and Post-Surgical Findings for Long-Term Neurocognitive and Behaviour Development Due to Posterior Fossa Pilocytic Astrocytomas: The Trend after 10 Years. Diagnostics 2021, 11, 1489. [CrossRef]

59. Piscione, P.J.; Bouffet, E.; Mabbott, D.J.; Shams, I.; Kulkarni, A.V. Physical functioning in pediatric survivors of childhood posterior fossa brain tumors. Neuro-Oncology 2014, 16, 147-155. [CrossRef]

60. Gunn, M.E.; Lähdesmäki, T.; Malila, N.; Arola, M.; Grönroos, M.; Matomäki, J.; Lähteenmäki, P.M. Late morbidity in long-term survivors of childhood brain tumors: A nationwide registry-based study in Finland. Neuro-Oncology 2015, 17, 747-756. [CrossRef]

61. Cohen-Cutler, S.; Olch, A.; Wong, K.; Malvar, J.; Sposto, R.; Kobierski, P.; Sura, A.; Constine, L.S.; Freyer, D.R. Surveillance for radiation-related late effects in childhood cancer survivors: The impact of using volumetric dosimetry. Cancer Med. 2021, 10, 905-913. [CrossRef] [PubMed]

62. Patel, S.K.; Mullins, W.A.; O'Neil, S.H.; Wilson, K. Neuropsychological differences between survivors of supratentorial and infratentorial brain tumours. J. Intellect. Disabil. Res. 2011, 55, 30-40. [CrossRef] [PubMed]

63. Toescu, S.M.; Samarth, G.; Layard Horsfall, H.; Issitt, R.; Margetts, B.; Phipps, K.P.; Jeelani, N.-U.-O.; Thompson, D.N.P.; Aquilina, K. Fourth ventricle tumors in children: Complications and influence of surgical approach. J. Neurosurg. Pediatr. 2020, $27,52-61$. [CrossRef] [PubMed] 